\title{
A necessidade de regulamento de interconexão para microgeração por máquinas síncronas e assíncronas
}

\author{
The need for interconnection regulation for microgeneration by \\ synchronous and asynchronous generators
}

\author{
Airam Tereza Zago Romcy Sausen ${ }^{1}$ \\ Alcedir Luis Finkler ${ }^{2}$ \\ Mauricio de Campos ${ }^{3}$ \\ Paulo Sérgio Sausen ${ }^{4}$
}

\section{RESUMO}

A geração de energia elétrica utilizando biogás em propriedades rurais tem sido amplamente pesquisada nos últimos anos. Diversos são os estudos demonstrando a viabilidade técnica, econômica e benefícios ao meio ambiente proporcionados por esse tipo de geração. Por outro lado, tanto produtores de diversas regiões do país como fabricantes que trabalham no desenvolvimento de equipamentos para geração voltados a essa demanda têm encontrado dificuldades para sua implementação devido a divergências em normas apresentadas por distribuidoras de energia elétrica. Neste artigo, é realizado um estudo comparativo entre as diferentes normas a que esse mercado está sujeito. Nesse sentido, são apresentadas as principais dificuldades encontradas, além de demonstrar a necessidade da formulação de uma norma mais objetiva, que permita a ampliação da utilização dessa fonte de energia e abertura desse mercado.

Palavras-chave: Biogás. Geração distribuída. Microgeração. Normas técnicas. Proteção anti-ilhamento.

1 Universidade Regional do Noroeste do Estado do Rio Grande do Sul - UNIJUI | airam@unijui.edu.br

2 Instituto Federal Farroupilha - Campus Santa Rosa | alcedir.finkler@iffarroupilha.edu.br

3 Universidade Regional do Noroeste do Estado do Rio Grande do Sul - UNIJUI | campos@unijui.edu.br

4 Universidade Regional do Noroeste do Estado do Rio Grande do Sul - UNIJUI | sausen@unijui.edu.br 


\section{A necessidade de regulamento de interconexão para}

microgeração por máquinas síncronas e assíncronas

The need for interconnection regulation for microgeneration by synchronous and asynchronous generators

\section{ABSTRACT}

The generation of electric energy using biogas in rural properties has been extensively researched in recent years. There are several studies showing the technical, economic feasibility and environmental benefits provided by this type of generation. On the other hand, both producers in different regions of the country and manufacturers working in the development of equipment for generation geared to this demand have encountered difficulties in their implementation due to divergences in standards presented by electricity distributors. In this article, a comparative study is carried out between the different standards to which this market is subject. In this sense, the main difficulties encountered are presented, in addition to demonstrating the need to formulate a more objective standard, which allows the expansion of the use of this energy source and the opening of this market.

Keywords: Biogas. Distributed Generation. Microgeneration. Standards. Anti-islanding protection. 


\section{Introdução}

A expansão industrial, o crescimento populacional e o desenvolvimento tecnológico, normalmente com objetivo de melhorar a qualidade de vida, apresentam, como contrapartida, um aumento na demanda por energia elétrica. O sistema hidráulico originalmente desenvolvido para produção de energia elétrica que se utiliza de grandes represas para armazenar água, conectado a longas linhas de transmissão, tem sido suplementado por sistemas de geração de pequeno porte, instalados próximos às unidades de consumo. Esse tipo de geração é chamado de geração distribuída (GD). Ackermann, Andersson e Söder (2001) destacam que existe uma tendência de investimentos nesses sistemas de geração. Neste trabalho, apresenta-se o estudo, desses autores, que descreve diversas terminologias utilizadas para se referir a esse tipo de geração. Os autores destacam, ainda, divergências entre os entendimentos de quais níveis de potência definiriam, por exemplo, micro, pequenas, médias ou grandes centrais geradoras. Em seus estudos, são relatadas diferenças entre normas de diversos países.

Nos Estados Unidos da América (EUA), até 1978, as empresas de distribuição de energia, a seu critério, poderiam ou não aceitar a conexão de sistemas de GD paralela a sua rede. Após esse período, criou-se a Lei de Política Regulatória de Utilidade Pública (Public Utility Regulatory Policies Act) (PURPA). Essa legislação, por sua vez, é semelhante à implantada no Brasil por meio da resolução 482 da Agência Nacional de Energia Elétrica (ANEEL) em 2012. Após a regulamentação por meio da PURPA, em 1978, nos EUA, consumidores que instalassem pequenos geradores poderiam conectar-se ao sistema interligado de potência, desde que atendidas as especificações técnicas. Ackermann, Andersson e Söder (2001) já descreviam o fato de geradores e operadores, normalmente, discordarem sobre o que atende ou não aos requisitos para conexão. No Brasil, a GD teve um salto após a resolução 482 da ANEEL, em 2012, com um foco à geração fotovoltáica. No entanto, existem alternativas que ainda podem ser exploradas. Nesse sentido, uma fonte que apresenta potencial, mas tem sido pouco utilizada, consiste na utilização de biogás como combustível para produção de energia elétrica.

No Brasil, a dificuldade para ingressar nesse mercado é a adequação aos requisitos das distribuidoras de energia. Este trabalho objetiva apresentar as divergências nas normas entre diferentes distribuidoras de energia e, a partir disso, sugere a implementação de uma normativa definindo um padrão a ser adotado por estas.

Por fim, este trabalho está organizado da seguinte forma: na seção 2, são apresentas as potencialidades para geração por meio de biogás; na seção 3 , são descritas vantagens e desvantagens com 0 uso da geração distribuída; na seção 4, são comparadas normas técnicas de diferentes companhias distribuidoras de energia e apontadas dificuldades encontradas por empreendedores que desejam investir nesse mercado; na seção 5, são discutidas propostas que poderiam solucionar as demandas aqui apresentadas.

\section{Geração distribuída e os sistemas de biodigestores}

Atualmente, estudos de viabilidade econômica para implementação de sistemas de geração de energia elétrica utilizando biodigestores em propriedades rurais, GD, são realizados, considerando todos os custos envolvidos para a construção dos biodigestores. Nesse sentido, cabe questionar se os custos dos biodigestores deveriam ou não estar incorporados no cálculo do custo para implementação desse tipo de geração.

Holm-nielsen, Al Seadi e Oleskowicz (2009) descrevem os impactos ambientais causados, em especial ao ar e à água, pelo despejo sem o devido tratamento dos dejetos de animais, entre eles, lixiviação de nutrientes da água e do solo, principalmente nitrogênio e fósforo, além de evaporação de amônia e contaminação patogênica. Destacam, ainda, a preocupação com a produção e emissão de 
gás metano proveniente desses resíduos, cujo impacto para o aquecimento global é aproximadamente vinte e três vezes maior comparado às emissões totais de $\mathrm{CO}_{2}$. Os autores citam também a utilização de biodigestores como uma solução adequada para a redução do potencial danoso desses resíduos. Por fim, em seu artigo, é apresentada a legislação europeia, onde são definidas as metodologias a serem implementados em propriedades rurais para dar o devido descarte aos dejetos animais, dentre as quais, o sistema de biodigestor destaca-se a partir da possibilidade da utilização do gás obtido para aquecimento ou produção de energia elétrica.

Em seu trabalho, Gadotti et al. (2018) reforçam que as propriedades rurais que atuam com a suinocultura exercem atividade considerada pelos órgãos ambientais como uma "atividade potencialmente causadora de degradação ambiental". Apresentam, ainda, que de acordo com a Lei 9.605/98, Lei de Crimes Ambientais, o produtor que não prever um tratamento adequado para os resíduos animais pode ser responsabilizado criminalmente por danos causados ao meio ambiente.

Nesse sentido, a geração de energia elétrica adotada em propriedades rurais poderia ser tratada como um incentivo, um bônus aos produtores que utilizam os biodigestores em suas propriedades rurais, uma vez que acabam por trazer benefícios ao meio ambiente. Em 2018, Bomfim (2018), em seu estudo, apresenta a capacidade de obtenção de biogás classificando por espécie animal, utilizando-se de biodigestores. 0 autor também desenvolve uma análise da capacidade de geração de energia elétrica considerando a produção de suínos por estados no Brasil, cuja capacidade de produção seria de 4.676,44 GWh/ano No caso do Rio Grande do Sul, a capacidade de geração de energia elétrica equivalente através de biodigestores, utilizando os rejeitos da suinocultura como matériaprima, é de 669,18 GWh/ano.

O estudo de viabilidade econômica do sistema de geração de energia elétrica em uma propriedade rural que possui em média 9.964 suínos foi realizado por Pasini et al. (2019). Nesse trabalho, é demonstrado que o retorno do investimento ocorre em cerca de três anos, considerando inclusive a construção dos biodigestores.

Cabe ressaltar, ainda, que para um melhor aproveitamento da energia elétrica gerada e uma maior contribuição do ponto de vista ambiental, essa deve ser injetada no sistema elétrico de potência de forma semelhante ao tratamento que é dado às fontes de energia fotovoltaica. Com isso, seria possível reduzir a demanda de energia fornecida por hidrelétricas, proporcionando uma maior flexibilidade em momentos de escassez de água.

Do ponto de vista social, esses investimentos tendem a melhorar a qualidade de vida dos produtores rurais, seja pela redução de odores e insetos atraídos por estes, seja pela redução dos custos de energia elétrica. A possibilidade de geração compartilhada, descrita no artigo 2, item VII, da resolução 482 de 2012 da ANEEL permite que diferentes unidades consumidoras realizarem a compensação do excedente de geração dentro de uma mesma área de concessão ou permissão. Esse incentivo, a partir de uma ampla divulgação e ações de esclarecimento, pode tornar esse tipo de geração um atrativo, inclusive, à instalação de pequenas indústrias em suas localidades.

Discutidos os benefícios ao meio ambiente e aos produtores rurais obtidos pela utilização da GD utilizando biodigestores conectados ao sistema elétrico de potência, cabe questionar quais são as dificuldades encontradas que podem impedir a sua implementação?

\section{Desafios da geração distribuída}

A utilização dos sistemas de GD não é recente, porém a legislação em torno dessa questão ainda não é clara. Nos Estados Unidos da América, a conexão de geradores paralelos à rede teve distintos altos e baixos ao longo da história. Por exemplo, ela foi de grande interesse de produtores rurais por volta de 1990 quando se permitiu a possibilidade de gerar energia com motores acionados a gás natural, porém, 
em contrapartida, em função da elevação dos custos do gás natural, acabou reprimindo a demanda. Uma nova fase surgiu por volta dos anos 2000, com foco nas energias renováveis (MOZINA, 2010).

Desde a publicação da PURPA, em 1978, existem preocupações quanto à segurança dos trabaIhadores que atuam em redes de energia elétrica. Uma delas consiste na possibilidade da rede estar energizada por meio de uma fonte geradora, conectada em alguma de suas extremidades durante manutenções, e dessa forma há um risco aos técnicos que atuam nessas redes. Assim, esforços no intuito de desenvolver uma normativa que garanta a segurança dos operadores vêm sendo aplicados desde então.

Gupta, Bhatia e Jain (2014) discorrem sobre o problema da segurança para equipes de manutenção que atuam no sistema de potência, devido à geração distribuída. Nesse sentido, uma contribuição ocorreu a partir da definição da IEEE 1547. A norma IEEE 1547 define que tão logo ocorra uma perda de conexão do sistema de geração distribuída com o sistema interligado de potência, essa geração deverá ser capaz de detectar a perda e automaticamente desconectar o gerador do sistema.

A norma IEEE 1547, ainda, classifica as unidades geradoras em três tipos sendo: geradores de indução, geradores síncronos e inversores. Os geradores de indução são equipamentos que produzem energia ativa quando conectados paralelos à rede e acelerados acima da velocidade síncrona. No entanto, para que esses gerem energia ativa, precisam absorver energia reativa da rede. Logo, esse equipamento, quando perde a conexão com a rede ou em caso de uma falta em seus terminais, interrompe a produção de energia em poucos segundos. Já os inversores são equipamentos projetados para seguir um perfil de tensão fornecido pela rede, logo, uma vez que é interropida a alimentação fornecida pelo sistema de potência, a geração é desconectada em poucos segundos. Dessa forma, a maior preocupação incide sobre os geradores síncronos, uma vez que estes possuem controladores de frequência e tensão que permitem que sigam operando mesmo com a perda de conexão com o sistema de potência. Em um extremo, eles podem ainda servir como fonte de energia reativa para geradores assíncronos ou produzir a tensão de referência necessária para que inversores sigam injetando energia na rede (MOZINA, 2010).

Campos et al. (2018) apresentam a modelagem de um sistema de geração distribuída com motor de combustão interna convertido a biogás. Descrevem como principal método utilizado para esse sistema de geração o uso de motores a diesel convertidos para biogás em conjunto com máquinas síncronas. Também afirmam que o sistema de geração de energia utilizado em unidades com biodigestores é predominantemente formado por geradores síncronos.

Assim, pode-se concluir que a conexão de GD paralelo ao sistema de potência apresenta diversas vantagens, porém requer atenção especial em alguns pontos. Um exemplo pode ser considerado, em que a abertura de um disjuntor em uma subestação poderia provocar a formação de um circuito composto por diversas cargas e geradores distribuídos conforme ilustrado na Figura 1. Nessa condição, mesmo ocorrendo a abertura do disjuntor na subestação, a rede poderia seguir energizada por meio de geradores distribuídos conectados a ela (MOZINA, 2010). 
Figura 1 - Geração distribuída operando em condição de ilhamento

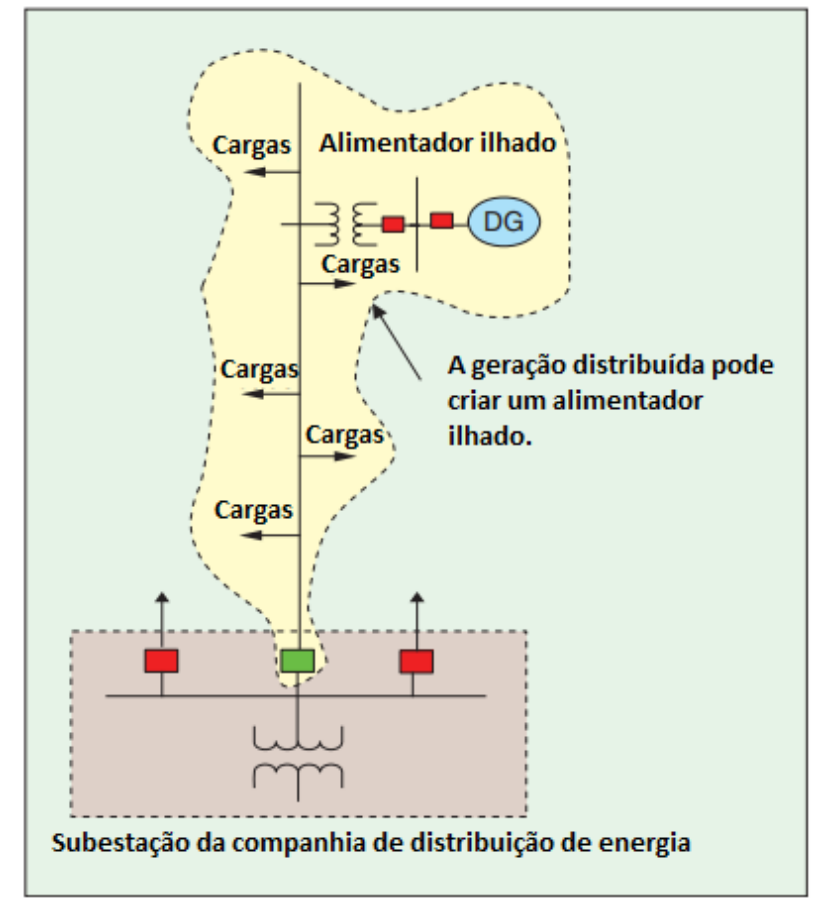

Fonte: Criado pelos autores com base em Mozina (2010)

Para além dos riscos à segurança dos trabalhadores que atuam no sistema de potência, a existência de GD conectados em paralelo com a rede apresenta outros pontos a serem observados. Ackermann, Andersson e Söder (2001) descrevem que, em sistemas de geração distribuída, os produtores podem injetar energia na rede de acordo com o preço de mercado, no entanto, isso não necessariamente coincide com as necessidades do sistema. Logo, uma vez que a geração distribuída atingisse valores significativos, poderia resultar em excedentes de geração. Zeineldin e Kirtley (2009) apresentam ainda que os religadores automáticos possuem tempo de atuação entre 500 milisegundos e 1 segundo, enquanto as normas, a exemplo da IEEE 1547, definem que, nas instalações com geração distribuída, deverá haver dispositivos de segurança com capacidade de detectar a perda de conexão com a rede e desconectar os geradores em tempo inferior a 2 segundos. Nesse sentido, durante uma falta que provocasse a abertura de religador, o gerador e o sistema de potência poderiam atuar com dinâmicas diferentes, fazendo com que, ao ocorrer uma tentativa de rearme do religador, o mesmo poderia estar operando fora de sincronismo, dessa forma, causando oscilações indesejadas ao sistema. Além disso, uma função dos religadores é, após a detecção de uma falta, causar uma breve interrupção no fornecimento de tensão, permitindo que, se possível, ocorra a extinção de um eventual arco elétrico. No entanto, com o uso da geração distribuída, a rede poderia continuar alimentada mesmo após a abertura do religador.

Além das dificuldades apresentadas, a inserção da geração distribuída introduz ainda problemas às empresas responsáveis pela distribuição de energia, dentre os quais é possível citar: alteração no sentido de circulação da corrente que afeta diretamente o dimensionamento dos equipamentos para proteção contra faltas (fluxo reverso), elevação do nível de curto-circuito colocando em risco projetos já existentes, entre outras. Em função dos pontos anteriormente descritos, Ackermann, Andersson e Söder (2001) descrevem o fato de geradores e operadores normalmente discordarem sobre o que atende ou não aos requisitos para conexão.

Dessa forma, de um lado, tem-se os produtores com a disponibilidade de energia a ser injetada na rede, associados a preocupações ambientais que justificam o uso de biodigestores para produção 
de energia elétrica, de outro, tem-se as distribuidoras com a preocupação quanto à segurança dos trabalhadores e proteção de suas instalações e equipamentos. Para organizar esse mercado, no Brasil, existe a Agência Nacional de Energia Elétrica, ANEEL onde entre os seus objetivos destaca-se a determinação de normas e procedimentos a serem seguidos por produtores e distribuidoras de energia elétrica. Como isso é feito?

\section{Legislação brasileira para microgeração distribuída}

A ANEEL foi instituída pela lei n 9.427/1996 e através da lei n 9.648/1998 foram estabelecidas as condições de acesso aos sistemas de distribuição de energia elétrica a partir de concessionário, permissionário ou autorizado, para microgeração distribuída. Nesse sentido, ainda, as principais normas e procedimentos relacionados são o Módulo 3 dos procedimentos de distribuição (PRODIST), e a resolução normativa 482 de 2012.

O PRODIST teve sua primeira versão aprovada em 2008. Atualmente, é composto por 11 módulos. Em seu Módulo 3, são estabelecidas as condições de acesso ao sistema de distribuição de energia. A seção 3.7 da revisão 7, que entrou em vigência em 01/06/2017, é dedicada ao acesso de micro e minigeração distribuída. No item 4 dessa seção, são definidos os requisitos necessários para o acesso de microgeração distribuída com potência instalada menor ou igual a 75 kW como (ANEEL, 2017):

I Elemento de desconexão: define a necessidade de haver um dispositivo que permita a acessada (companhia distribuidora de energia em que o sistema de geração distribuída está conectado) realizar a desconexão física da rede em caso de necessidade de manutenção.

I Elemento de interrupção: corresponde ao componente do sistema que realiza a interrupção da geração em caso de atuação de alguma das proteções.

I Proteção de sub e sobre tensão e proteção de sub e sobre frequência: a norma deixa claro que não é necessário equipamento específico, podendo ser realizada por algum relé eletromecânico capaz de atuar os elementos de interrupção.

I Relé de sincronismo: de forma semelhante ao item anterior, a norma define que não é necessário relé de sincronismo específico, porém exige que exista um sistema eletromecânico capaz de identificar o sincronismo entre a tensão produzida pelo gerador e a tensão produzida pela rede e capaz de operar o elemento de interrupção.

I Anti-ilhamento: é ressaltado na norma que é vedada a conexão do sistema de geração ao sistema da distribuidora durante interrupções de fornecimento. Essa proteção deverá garantir a desconexão física nessa situação. Porém não é definido na norma qual o tipo de equipamento que deve ser utilizado.

A resolução 482 de 2012 da ANEEL, além de definir o modelo em que será realizada a compensação da energia produzida, define as condições para que os produtores possam se conectar ao sistema de distribuição de energia. Limita a classificação de microgeração distribuída à potência instalada menor ou igual a $75 \mathrm{~kW} / \mathrm{h}$. Em seu artigo terceiro, define que é responsabilidade das distribuidoras elaborar as normas técnicas que serão utilizadas para tratar do acesso a microgeração distribuída. Estabelece, ainda, que tais normas deverão observar como referência o PRODIST, normas técnicas brasileiras e, de forma complementar, as normas técnicas internacionais (ANEEL, 2012). O PRODIST traz uma orientação quanto às proteções necessárias para geração distribuída e, em sua sétima revisão, na seção 3.7, item 4.6, onde define os requisitos de projeto, especifica que (ANEEL, 2017, p. 65):

$A$ acessada pode propor proteções adicionais, desde que justificadas tecnicamente, em função de características específicas do sistema de distribuição acessado, sem custos para microgeração distribuída. 
Estariam as distribuidoras de energia seguindo essa orientação? Poderiam elas definir proteções diferentes das especificadas no Módulo 3 do PRODIST? Caso tais proteções fossem solicitadas por determinadas distribuidoras, quem deveria arcar com os custos destas? Como estão organizadas atualmente as normas criadas pelas distribuidoras?

A Companhia Paulista de Força e Luz (CPFL), em atendimento ao artigo terceiro da resolução 482 de 2012 da ANEEL, disponibiliza a norma técnica GED 15303 sob o título "Conexão de Micro e Minigeração Distribuída sob Sistema de Compensação de Energia Elétrica". Em sua versão 1.5, publicada em 11/06/2019, no item 5.30, define que:

- Toda central de microgeração distribuída conectada na rede de baixa tensão (BT) da $\mathrm{CPFL}$, independentemente da quantidade de fases e da potência que pode ser gerada, deverá sê-lo necessariamente por intermédio de inversores eletrônicos, qualquer que seja a fonte primária da energia (CPFL, 2019, p. 24).

Essa solicitação dificulta a implementação de geradores distribuídos que utilizam os sistemas de biodigestores para obtenção de biogás e uso desses para acionamento de motores a combustão com a geração sendo realizada por máquinas síncronas ou máquinas assíncronas (CPFL, 2019).

No Rio Grande do Sul, a Federação das Cooperativas de Energia, Telefonia e Desenvolvimento Rural do Rio Grande do Sul (FECOERGS), é formada por 23 cooperativas de eletrificação rural. Em 2002, a FECOERGS definiu, em seu planejamento estratégico, a necessidade de adoção de uma padronização das atividades de distribuição de energia elétrica. Atualmente, as cooperativas associadas seguem as normativas disponíveis no site da FECOERGS. Para a microgeração distribuída, em atendimento ao artigo terceiro da resolução 482 de 2012 da ANEEL, é disponibilizada a normativa OTD 035.01.08. Em sua revisão de 28/06/2018, no item 7.4, descreve os requisitos mínimos de proteção necessários para conexão de microgeração distriuída ao sistema de baixa tensão. Relaciona ainda as funções ANSI de cada proteção conforme tabela 1. Em seu anexo XI, fornece os valores a serem ajustados em cada uma das funções ANSI, com exceção à proteção 78, a qual faz referência apenas à necessidade de cessar fornecimento de energia em até 2 segundos ao detectar a perda de conexão com a rede, deixando a escolha do método de detecção e a definição dos parâmetros sob responsabilidade do projetista (FECOERGS, 2019).

Tabela 1 - Requisitos mínimos de proteção para microgeração BT

\begin{tabular}{l|c}
\hline Equipamento & Função ANSI \\
\hline Proteção de Subtensão & 27 \\
\hline Proteção de Sobretensão & 59 \\
\hline Proteção de Sobrefrequência & 810 \\
\hline Proteção de Subrefrequência & $81 U$ \\
\hline Relé de Sincronismo & 25 \\
\hline Proteção Anti-ilhamento & 78 \\
\hline
\end{tabular}

Fonte: Adaptado de FECOERGS (2019)

A Companhia Paranaense de Energia (COPEL), em atendimento ao artigo terceiro da resolução 482 de 2012 da ANEEL, disponibiliza a normativa NTC 905200. Em sua revisão de outubro/2018, no item 5, define que a geração poderá ocorrer por meio de máquinas assíncronas para potência de até $300 \mathrm{~kW}$, logo, possibilitando o uso desse tipo de equipamento para microgeração distribuída. Define que, para estas, o fator de potência deverá ser mantido superior a 0,92. Descreve também que a correção do fator de potência para esse tipo de geração deverá ser realizada por meio de banco de capacitores automático, com o objetivo de evitar a possibilidade de ilhamento. É definido que a capacidade do banco de capacitores não deverá ultrapassar 75\% da potência reativa máxima do gerador de indução. 
Apresenta, ainda, conforme figura 2, o esquema unifilar para conexão de microgeção distribuída em redes de baixa tensão, BT (COPEL, 2016).

Para proteção do sistema, são solicitadas as funções ANSI 25, 27, 59, 78, 81U/0 e 81df/dt. 0 documento, em seu item 5.2.1d, justifica a importância dos relés para detecção de ilhamento e define a necessidade de implementação dessa função pelos métodos de taxa de variação da frequência (ANSI $81 \mathrm{df} / \mathrm{dt}$ ), e relé de salto de vetor (ANSI 78). São fornecidos os valores de ajustes para as funções ANSI $27,59,81 \mathrm{U} / 0$, porém fica a cargo do projetista a especificação dos valores a serem ajustados para as funções ANSI 78 e 81df/dt (COPEL, 2016).

Figura 2 - Esquema unifilar típico para geração em BT

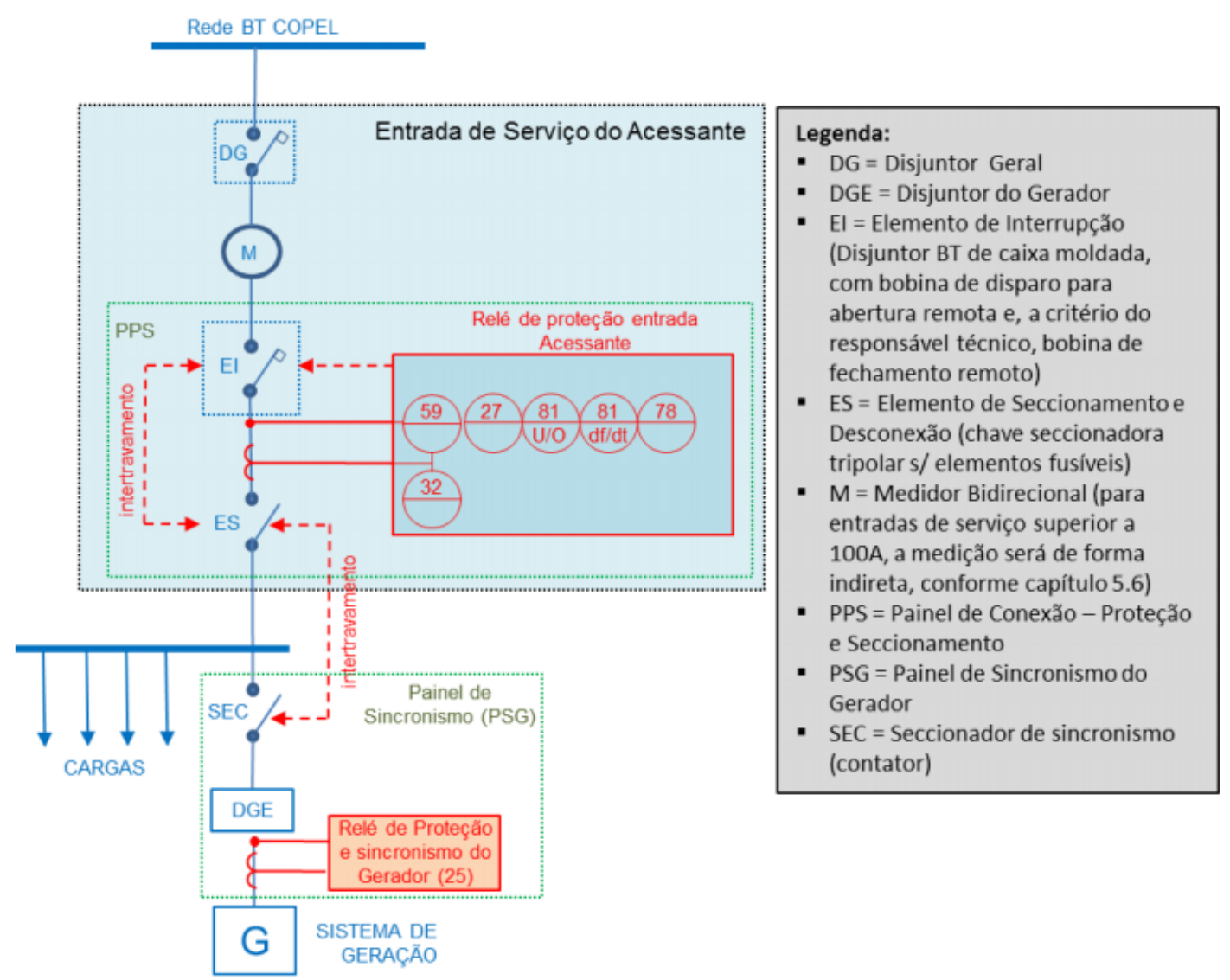

Fonte: COPEL (2016)

A norma NTC 905200, disponibilizada pela COPEL, delimita o tempo mínimo para que o gerador possa se reconectar à rede após uma falta de energia de 2 minutos. Além disso, descreve as licenças de operação que serão solicitadas para cada tipo de geração. Por exemplo, para geração realizada a partir da energia liberada na forma de calor, por meio da combustão de algum combustível renovável ou não renovável, será exigida licença ambiental prévia, licença de instalação e também licença de operação (COPEL, 2016).

Em uma comparação entre os requisitos para conexão com a rede das três companhias descritas na secção 4, é possível observar que, a exemplo da COPEL, sua norma técnica está organizada de forma detalhada e facilita significativamente a interligação de microgeradores distribuídos à sua rede, fazendo uma ressalva à carência quanto ao desenvolvimento de um método, procedimento ou forma de cálculo que permita ao projetista determinar com maior facilidade os parâmetros a serem ajustados no relé de taxa de variação da frequência (ANSI 81df/dt) e relé de salto de vetor (ANSI 78). Por outro 
lado, a CPFL coloca como limitação a necessidade de que a conexão seja realizada por meio de inversor de frequência. Logo, há severas divergências entre os equipamentos exigidos por ambas companhias.

Para empresas que buscam desenvolver um produto, com o objetivo de prover algum tipo de solução para este segmento e o comercializar em nível nacional, essas divergências entre as normas das distribuidoras tornam-se um obstáculo. Como Ackermann, Andersson e Söder (2001) já descreviam em seu trabalho, o fato de geradores e operadores normalmente discordarem sobre o que atende ou não aos requisitos para conexão, cabe questionar se não existe a necessidade de que a ANEEL apresente uma normativa mais específica, mais detalhada, para conexão de microgeração, principalmente, por meio de máquinas síncronas e assíncronas.

\section{Considerações}

No presente artigo, são descritas as potencialidades da geração distribuída através de Biogás, sua viabilidade e importância ambiental. É realizada uma análise das vantagens no uso dessa geração pelos produtores, porém, quando abordada a legislação, fica evidente que a falta de uma normatização específica neste setor impõe dificuldades para a sua expansão. São apresentadas as dificuldades encontradas em algumas distribuidoras na gestão dessa geração e as normas que regulamentam esse tipo de geração, bem como as divergências entre normas de diferentes companhias distribuidoras em relação aos dispositivos de proteção necessários para conexão da geração distribuída. É realizada uma reflexão sobre a necessidade de uma revisão dessas normas com intuito de viabilizar o investimento nesse setor.

\section{Agradecimentos}

Ao Instituto Federal Farroupilha - Campus Santa Rosa, pelo apoio e incentivo à qualificação profissional e, em especial, ao Programa Institucional de Incentivo à Qualificação Profissional dos Servidores do IFFar (PIIQP).

\section{Referências}

ACKERMANN, T.; ANDERSSON, G.; SÖDER, L. Distributed generation: a definition. Electric power systems research, v. 57, n. 3, p. 195-204, 2001.

AGÊNCIA NACIONAL DE ENERGIA ELÉTRICA (ANEEL). Procedimentos de Distribuição de Energia Elétrica no Sistema Elétrico Nacional (PRODIST): Módulo 3 - Acesso ao Sistema de Distribuição. 2017. Disponível em https://www.aneel.gov.br/modulo-3. Acesso em: 18 de julho de 2020.

AGÊNCIA NACIONAL DE ENERGIA ELÉTRICA (ANEEL). Ministério de Minas e Energia. Resolução Normativa $n^{\circ}$ 482, de 17 de abril de 2012. Estabelece as condições gerais para o acesso de microgeração e minigeração distribuída aos sistemas de distribuição de energia elétrica, o sistema de compensação de energia elétrica, e dá outras providências. Disponível em: http://www.aneel.gov.br/cedoc/ren2012482.pdf. Acesso em: 18 de julho de 2020.

BOMFIM, G. R. et al. Estimativa da produção de biogás de origem suína no brasil. Revista Engenharia em Ação UniToledo, v. 3, n. 2, p. 164-179, 2018.

CAMPOS, B. A. P. et al. Biogas fueled internal combustion engine Mean Value Model for distributed generation. In: SIMPÓSIO BRASILEIRO DE SISTEMAS ELETRICOS (SBSE), Instituto de Engenheiros Eletricistas e Eletrônicos, Niteroi, Anais [...], p. 1-6, 2018. 
COMPANHIA PARANAENSE DE ENERGIA (COPEL). Norma Técnica 905200: acesso de micro e minigeração distribuída ao sistema da COPEL (com compensação de energia). Disponível em: https://www.copel.com/hpcopel/ root/sitearquivos2.nsf/verdocatual/E59DF9E94B635F678325831D0047F719/\$FILE/NTC905200_Rev04102018. pdf. Acesso em: 18 de julho de 2020.

COMPANHIA PAULISTA DE FORÇA E LUZ (CPFL). Norma Técnica GED 15303: Conexão de Micro e Minigeração Distribuída sob Sistema de Compensação de Energia Elétrica. Disponível em: http://sites.cpfl.com.br/ documentos-tecnicos/GED-15303.pdf. Acesso em: 18 de julho de 2019.

FEDERAÇÃO das Cooperativas de Energia, Telefonia e Desenvolvimento Rural do Rio Grande do Sul (FECOERGS). Orientação Técnica OTD 035.01.08: requisitos técnicos para conexão de micro e minigeração ao sistema de distribuição. Disponível em: https://www.fecoergs.com.br/anexos/035-01-08_Requisitos_Tecnicos_para_conexao_da_micro_e_minigeracao.pdf? $=5$. Acesso em: 18 de julho de 2019.

GADOTTI, G. I. et al. Biodigestores para tratamento de dejetos suínos e alternativas para o desenvolvimento sustentável da propriedade. Colloquium Agrariae, v. 14, n. 1, p. 120-128, 2018.

GUPTA, P.; BHATIA, R. S.; JAIN, D. K. Average absolute frequency deviation value based active islanding detection technique. IEEE Transactions on Smart Grid, v. 6, n. 1, p. 26-35, 2014.

HOLM-NIELSEN, J.B.; AL SEADI T.; OLESKOWICZ P.P., The future of anaerobic digestion and biogas utilization. Bioresource Technology, v. 100, n. 22, p. 5478-5484, 2009.

MOZINA, Charles J. Impact of green power distributed generation. IEEE Industry Applications Magazine, v. 16, n. 4, p. 55-62, 2010.

PASINI, F. et al. Viabilidade de implantação de um biodigestor em uma granja de suínos. Holos Environment, v. 19, n. 1, p. 60-69, 2019.

ZEINELDIN, H. H.; KIRTLEY, J. L. A simple technique for islanding detection with negligible nondetection zone. IEEE Transactions on Power Delivery, v. 24, n. 2, p. 779-786, 2009. 